\title{
Successful endovascular embolization of a giant splenic artery pseudoaneurysm secondary to a huge pancreatic pseudocyst with concomitant spleen invasion
}

\author{
Antonio Borzelli ${ }^{1 A, B, E}$, Francesco Amodio ${ }^{1 B, D}$, Francesco Pane ${ }^{2 F}$, Milena Coppola ${ }^{2 A}$, Mattia Silvestre ${ }^{1 B}$, \\ Marco Di Serafino ${ }^{3 B}$, Fabio Corvino ${ }^{1 E}$, Francesco Giurazza ${ }^{1 A, D, E}$, Raffaella Niola ${ }^{10, E}$ \\ 'Department of Vascular and Interventional Radiology, AORN “A. Cardarelli", Napoli, Italy \\ 2Department of Radiology, AORN "S.G. Moscati", Avellino, Italy \\ 3Department of General and Emergency Radiology, AORN “A. Cardarelli”, Napoli, Italy
}

\section{Abstract}

Pseudoaneurysms of the pancreatic and peripancreatic arteries is a well-known complication of chronic or necrotizing pancreatitis due to proteolytic enzymatic digestion of the arterial wall. A major part of peripancreatic pseudoaneurysms involve the splenic artery, but any peripancreatic artery may be involved and bleed. They are potentially life threatening for patients, due to spontaneous intraperitoneal rupture, rupture and fistulization into the surrounding organs, or fistulization into the pancreatic duct. Small ones are usually asymptomatic and are often diagnosed incidentally, while giant $(>5 \mathrm{~cm})$ aneurysms and pseudoaneurysms are symptomatic and may be detected as a pulsatile mass in the upper-left quadrant or epigastrium. Imaging plays a key role in the identification of splenic artery aneurysms and pseudoaneurysms, while angiography still represents the gold standard for the diagnosis, although nowadays it plays a prominent role in treatment. Treatment of splenic artery pseudoaneurysms is mandatory because of the high probability of rupture, with a mortality rate of up to $90 \%$. The gold standard treatment is represented by surgery, with a mortality rate between $16 \%$ and $50 \%$. In recent years the endovascular approach has proven to be an effective alternative treatment for splenic artery pseudoaneurysms, and it is currently the method of choice. In this article, we present the case of a ant pseudoaneurysm of the splenic artery due to huge pseudocysts in a young alcoholic patient with recurrent and

chronic pancreatitis, complicated by fistulization and invasion of spleen parenchyma and arteriovenous fistula.

Key words: pseudoaneurysm, chronic pancreatitis, endovascular embolization, splenic artery.

\section{Introduction}

The splenic artery is the most common visceral artery affected by pseudoaneurysms, followed by the gastroduodenal and the pancreaticoduodenal arteries [1-3].
Pseudoaneurysms of the pancreatic and peripancreatic arteries is a well-known complication of chronic or necrotizing pancreatitis due to proteolytic enzymatic digestion of the arterial wall - the most important enzyme in this digestion process of the arterial wall is trypsin [4-6]. Less frequently, they can occur after trauma, probably due to rapid deceleration with consequent damage to the intima and elastic lamina $[1,2,7]$. They are potentially life threatening for patients, due to spontaneous intraperitoneal rupture, rupture and fistulization into the surrounding and hollow organs, or fistulization into the pan-

Correspondence address:

Antonio Borzelli, Department of Vascular and Interventional Radiology, AORN “A. Cardarelli”, Via A.Cardarelli 9, 80137, Napoli, Italy, e-mail: antonio.borzelli@libero.it Authors' contribution:

A Study design · B Data collection · C Statistical analysis · D Data interpretation - E Manuscript preparation · F Literature search · G Funds collection 
creatic duct $[8,9]$. Small ones are usually asymptomatic and are often diagnosed incidentally; in contrast, giant $(>5 \mathrm{~cm})$ aneurysms and pseudoaneurysms are symptomatic and may be detected as a pulsatile mass in the upper left quadrant or epigastrium, with referred epigastric and left upper quadrant abdominal pain, but also with feeling of fullness, anorexia, nausea, and vomiting $[8,10]$. Imaging techniques such as ultrasound, Doppler sonography, and computed tomography (CT) play a key role in the identification of splenic artery aneurysms and pseudoaneurysms, while angiography still represents the gold standard for the diagnosis, but nowadays it has a prominent role for treatment, with the possibility to perform an endovascular embolization $[1,11]$. Treatment of splenic artery pseudoaneurysms is mandatory because of the high probability of rupture with intra- or retroperitoneal haemorrhage or gastrointestinal bleeding, with a mortality rate of up to $90 \%[1,3]$. Historically, these lesions have been managed by open or laparoscopic surgical approach, with a mortality rate between 16 and $50 \%[1,2,12,13]$. In recent years, endovascular techniques have proven to be an effective alternative treatment for splenic artery pseudoaneurysms, and the endovascular approach is nowadays the method of choice, although the reported failure rate in literature is between 10 and $15 \%[1,2,12,13]$. In this article, we present the case of a giant pseudoaneurysm of the splenic artery due to huge pseudocysts in a young alcoholic patient with recurrent and chronic pancreatitis, complicated by fistulization and invasion of spleen parenchyma and arteriovenous fistula.
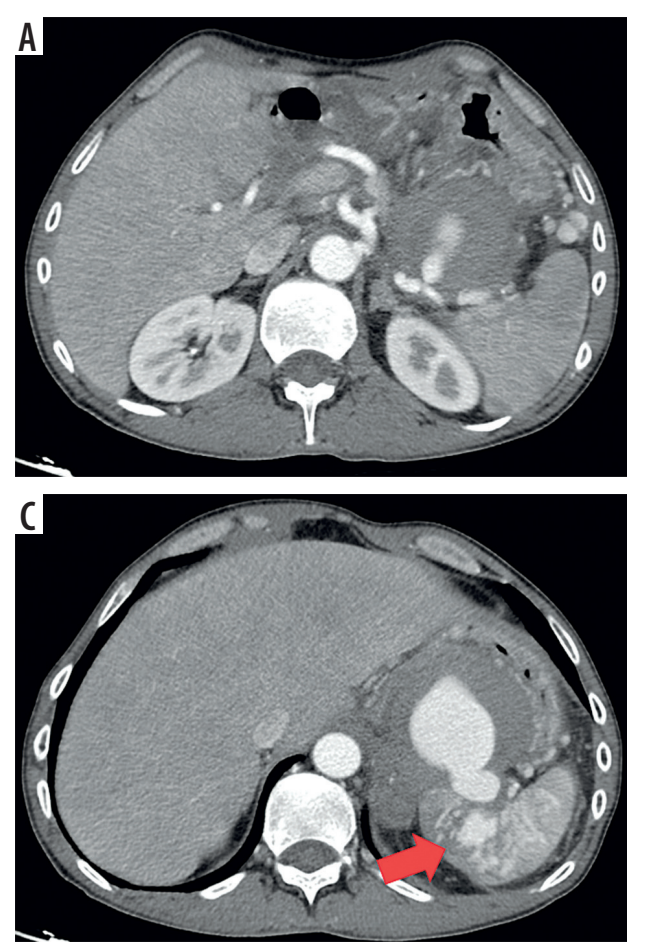

Figure 1. Computed tomography (CT) scan showing, in arterial phase, a giant pseudoaneurysm of the splenic artery (A, B) secondary to a huge pseudocyst (C) with concomitant invasion of upper pole spleen parenchyma and arterio-venous fistulas (D) (red arrows)

\section{Case presentations}

A 45-year-old man was affected by left upper quadrant abdominal pain and a feeling of fullness and pulsation. His medical history was notable for alcoholism, with previous recurrent acute pancreatitis, and smoking; there was no history of hypertension, trauma, fever, or abdominal or chest infections. His general physical examination was unremarkable, and his laboratory tests were normal. A contrast-enhanced CT scan revealed the presence of 2 huge pseudocysts, and, into the major one, localized close to pancreatic tail, the presence of a giant pseudoaneurysm of the splenic artery, with a maximum diameter of $80 \mathrm{~mm}$, with associated spleen invasion and concomitant arterio-venous fistulas (Figures 1 and 2A-B). Moreover, the splenic vein was occluded with development of many renal, mesenteric, and omental collaterals (Figure 2C-D). In the angiography suite, a selective celiac trunk and then super-selective splenic artery angiography were performed through a standard percutaneous right transfemoral approach, confirming the CT findings and showing the pseudoaneurysm's origin distal to the great pancreatic artery ostium (Figure 3A). An endovascular exclusion of the pseudoaneurysm was performed by transcatheter embolization with multiple microcoils (Nester, Cook-Medical, Bloomington, Indiana) released distal and proximal to pseudoaneurysm's origin ostium, with coaxial technique, employing a microcatheter (Progreat 2.7 Fr, Terumo, Shibuya-ku, Tokyo, Japan) for super-selective catheterisation, occluding the entire spleen artery segment involved but preserving the great pancreatic artery's ostium
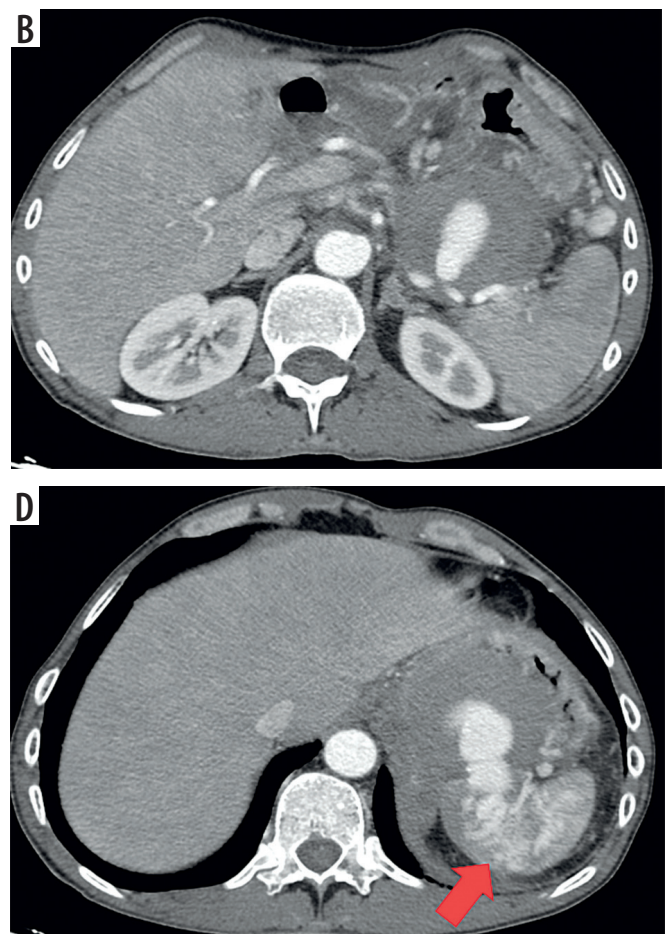

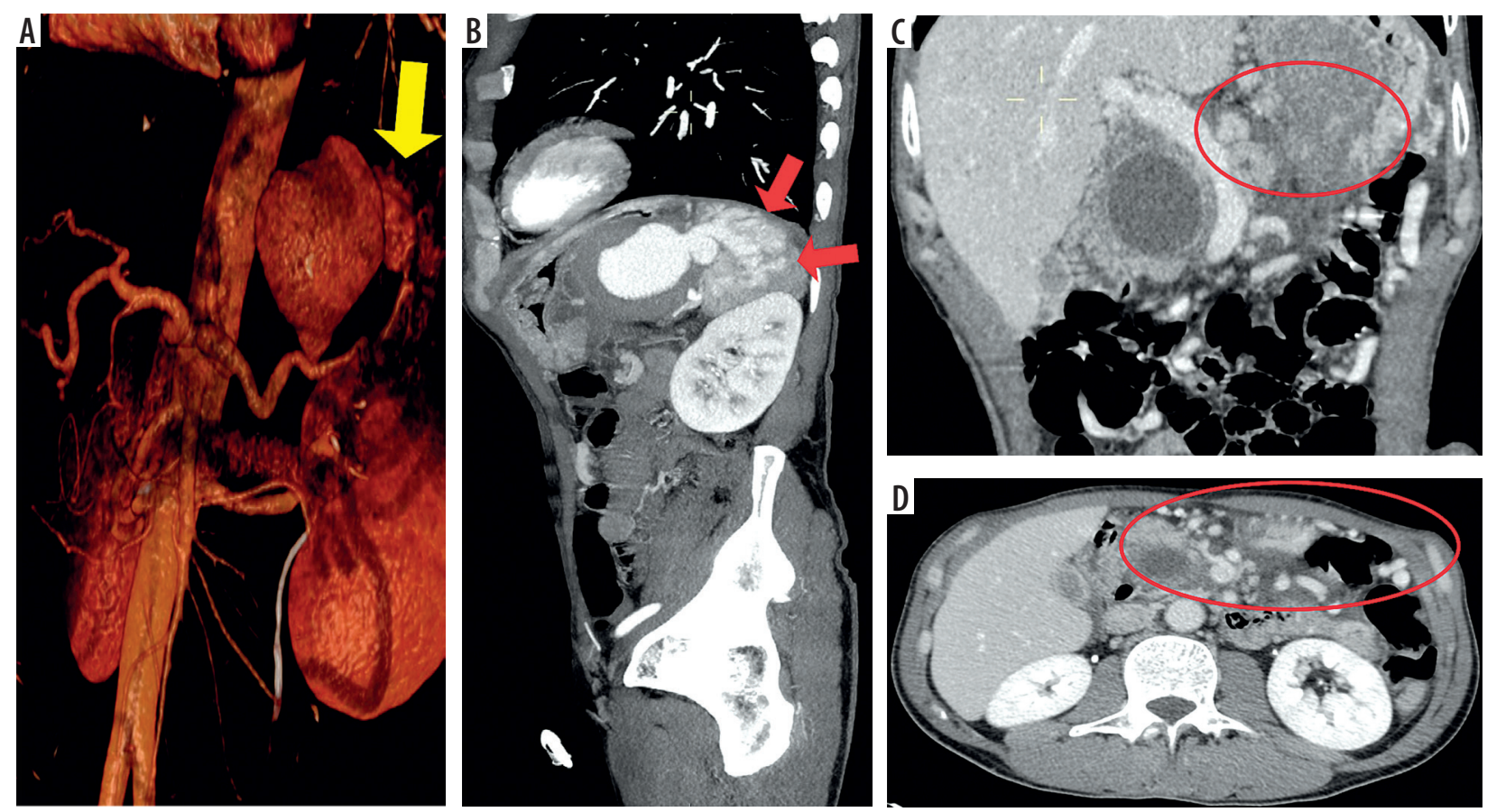

Figure 2. Computed tomography (CT) scan 3D-VR (A) and MIP sagittal (B) reconstructions showing a huge pseudoaneurysm of the splenic artery secondary to pancreatic pseudocyst and concomitant invasion of spleen (yellow arrow) and arterio-venous fistulas (red arrows); (T coronal (C) and axial (D) scan in portal venous phase showing chronic occlusion of splenic vein (C) (red circle) and concomitant development of many mesenteric and omental venous collaterals (D) (red oval)
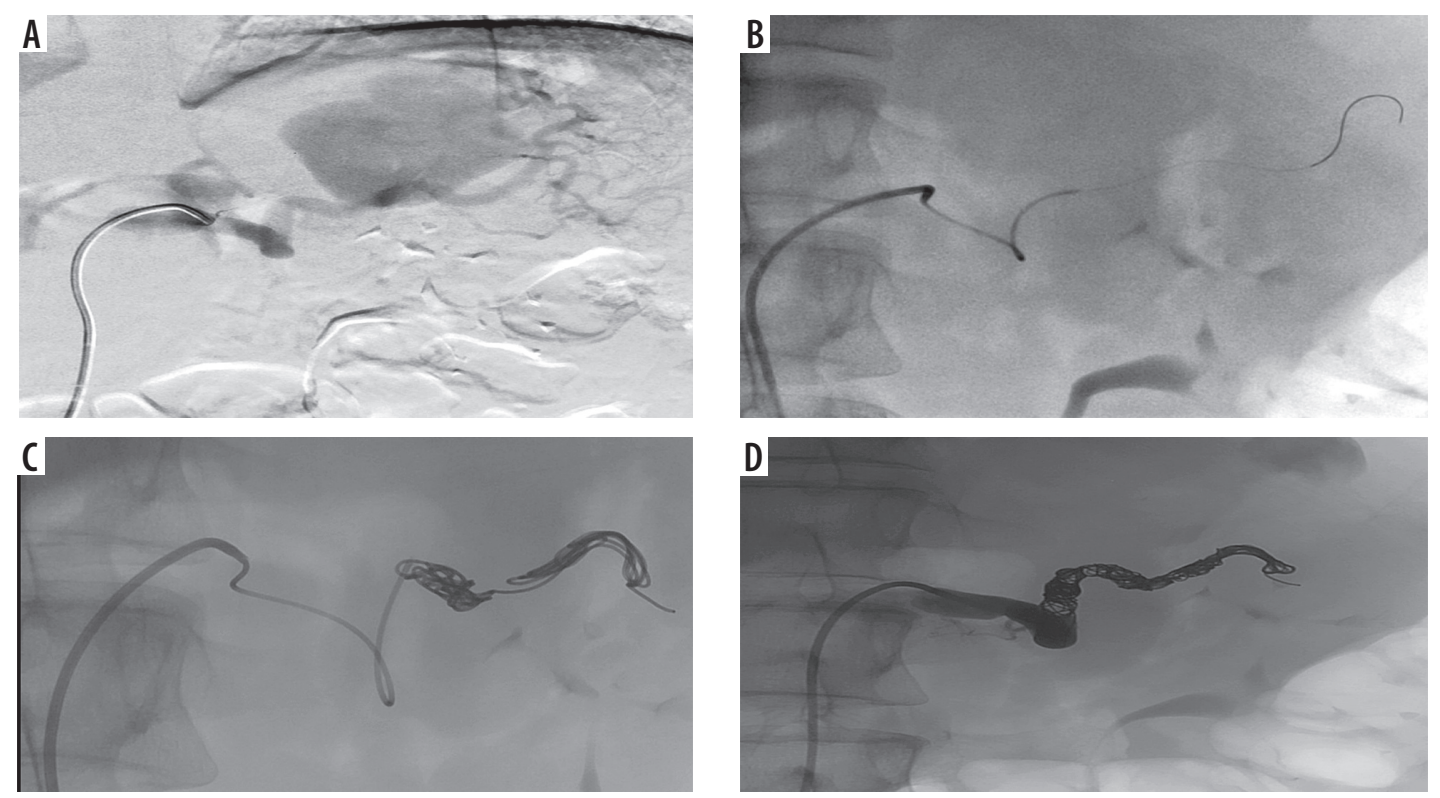

Figure 3. Digital angiography. Selective angiography of splenic artery confirming a giant pseudoaneurysm (A) and selective catheterization, employing a microcatheter, of distal portion of splenic artery (B). Endovascular embolization releasing multiple metallic microcoils into the in-flow and out-flow tracts of the giant pseudoaneurysm (C). Final angiographic control confirming successful endovascular exclusion of the pseudoaneurysm with opacification of the great pancreatic artery (D)

(Figure 3B-C). The final angiographic control showed successful and complete exclusion of the pseudoaneurysmal sac and of the intrasplenic arterio-venous fistula, with patency of the great pancreatic artery (Figure 3D). The patient was asymptomatic after the procedure and was discharged 3 days later, when a contrast-enhanced CT scan confirmed the technical success, with evidence of only minimum and neglectable ischaemic portions of splenic parenchyma, which was regularly vascularized due to collateral arterial supply to the organ coming from the gastric arteries and gastroduodenal arch through the left gastroepiploic artery (Figure 4). Six months after treatment he is still asymptomatic, and the endovascular embolization has proven to be effective.

\section{Discussion}



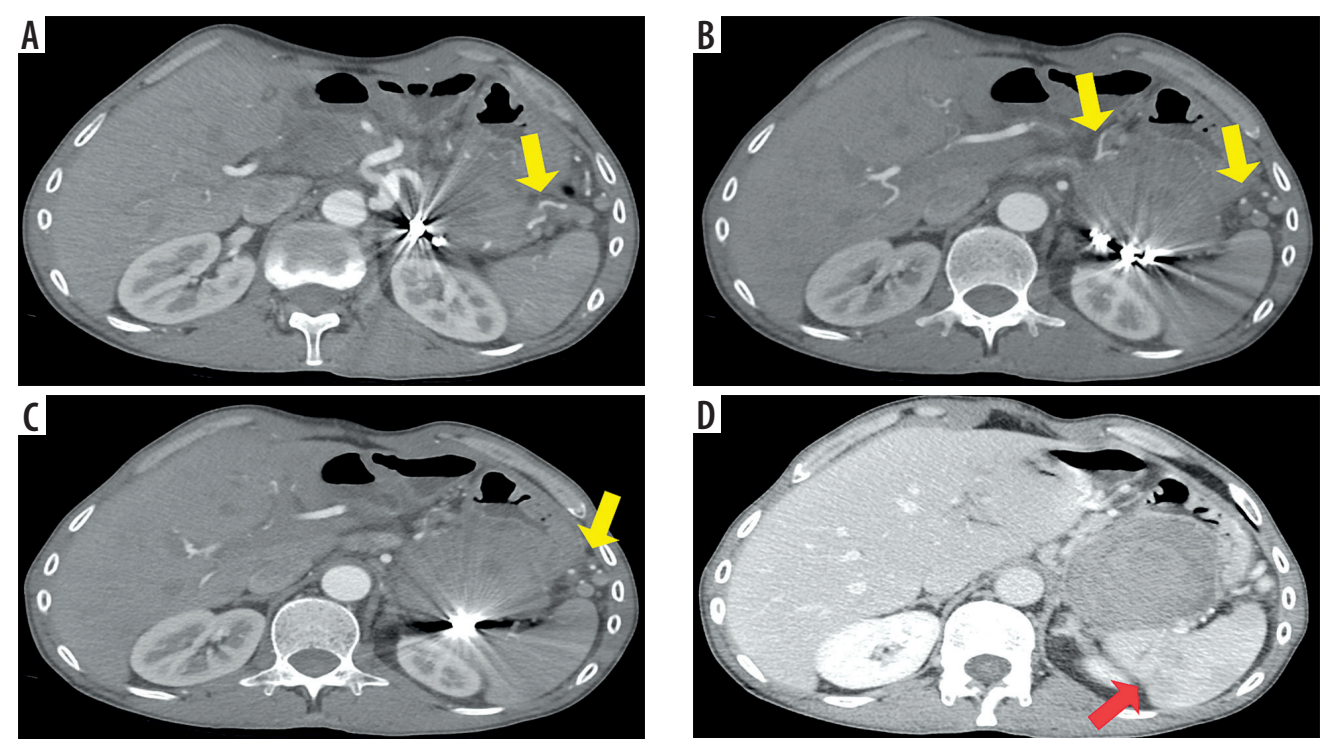

Figure 4. Computed tomography (CT) scan performed 3 days after the procedure, confirming in arterial phase (A-C) successful endovascular embolization with multiple metallic microcoils and no opacification of the giant pseudoaneurysm, with evidence of collateral arterial supply towards the spleen (yellow arrows); portal venous phase (D) confirming no opacification of the pseudoaneurysmal sac and subsequent minimum ischaemic areas in the context of spleen parenchyma (red arrow)

Splenic artery aneurysms represent between 50\% and 70\% of all visceral artery aneurysms and are the third most frequent intrabdominal aneurysms after those of the abdominal aorta and iliac arteries $[8,10,15]$. Various studies of autopsies and angiographies report rates of incidence at $0.01-0.20 \%$ and $0.78-0.80 \%$, respectively $[8,9,16]$. However, splenic artery aneurysms have been shown to be 4 times more frequent in women and most commonly identified in people older than 50 years $[8,9,14]$. They can be classified histopathologically as either true aneurysms (involving intima, media, and adventitia layers of the artery wall) or pseudoaneurysms (not involving all 3 artery wall layers) $[8,17]$. They usually measure $2.1 \mathrm{~cm}$ in diameter at the time of diagnosis, rarely exceeding $3 \mathrm{~cm}$; if their maximum diameter is $>5 \mathrm{~cm}$, they are generally defined as giant $[8,9,16]$. Chronic and acute pancreatitis represents the primary risk factor for splenic artery pseudoaneurysms, together with the presence of pancreatic pseudocysts and occurrence of abdominal trauma $[8,10,18]$. Pseudoaneurysms are due to the disintegration of elastin fibres of the artery wall by pancreatic enzymes with consequent arterial wall destruction $[8,18]$. In our case, in fact, the giant pseudoaneurysm was due to a huge pseudocyst for chronic pancreatitis that caused a laceration of the splenic artery. Haemorrhage due to wall erosion and pseudoaneurysm arising in the division branches of the celiac trunk is not frequent but represents a catastrophic complication of acute necrotizing pancreatitis $[4,19]$. A major part of peripancreatic pseudoaneurysms involve the splenic artery, as in our case, but any peripancreatic artery may be involved and bleed. Alcoholism represents the first cause of necrotizing pancreatitis, and pseudoaneurysm of a celiac branch constitutes a major cause of death in affected patients. In such patients, in fact, the ero- sion of an important artery can be a likely source of gastrointestinal bleeding. These patients are often very debilitated and, in this way, they present a high surgical risk [4]. Imaging plays a key role in the detection of these conditions, and the main diagnostic tools are ultrasound (US), Doppler US, contrast enhanced US (CEUS), CT, and magnetic resonance imaging (MRI) [8,16,20-24]. Doppler US is the first choice for pregnant patients, and it can be effective and sufficient, depicting precisely the location and the morphology of the false aneurysm in superficial anatomical districts, but it has a lower accuracy in abdomen, because of intestinal bloating, and has many limitations in emergency settings if the patient is not collaborating, while CEUS was recently proven to be a powerful new tool to detect false aneurysms, both for the first diagnosis, in patients with a clinical suspicion, and for follow-up after treatment, allowing a faster, easier, cheaper, repeatable, and above all, valid and effective radiation-free imaging. CT angiography, however, represents the gold standard for diagnosis, showing the typical aneurysm body in the arterial phase, as demonstrated in our case [8,10,17,20-25]. MRI proved to be more sensitive and specific, but it is contraindicated for patients with pacemakers and metal prostheses, it is unsuitable for claustrophobic and respiratory distressed patients, especially considering the prolonged time of execution, and above all, its availability is still limited in emergency settings $[8,10]$. Treatment is recommended for all symptomatic splenic artery aneurysms, regardless of the diameter, and for all asymptomatic patients in whom the aneurysmal sac diameter is $>2 \mathrm{~cm}$, due to their high risk of rupture associated with high morbidity and mortality rates, and for those who are pregnant or fertile, who are affected by portal vein hypertension or are candidates for liver transplantation $[8-10,25,26]$. In 
contrast, the rupture risk for pseudoaneurysms is not related to their diameter, and all pseudoaneurysms should be treated [8,27-29]. In fact, owing to their thin and weak walls, they can easily rupture and bleed spontaneously; in this way, the common clinical presentation is represented by gastrointestinal bleeding, and bleeding into a pseudocyst is the most common presentation of pancreatitisrelated pseudoaneurysms $[4,6]$. Spontaneous rupture and fistulization into neighbouring organs are the most important life-threatening complications of giant splenic artery pseudoaneurysms [8]. These are exactly the findings depicted in our patient: a giant pseudoaneurysm with bleeding into a pseudocyst and subsequent invasion of the upper splenic pole associated with venous fistulization. Surgery represents the gold standard therapy, and the most common approach has been suture ligation of the bleeding point in the wall of the pancreatic pseudocyst. However, this approach is characterized by high incidence of rebleeding and reoperation, and surgically it would be better to identify the involved vessel and to ligate it proximally and distally to the pseudoaneurysm $[4,19]$. However, in the last few years, surgery has been replaced by transcatheter endovascular embolization due to its low morbidity and mortality and high success rate $[25,30]$. In fact, in recent years transcatheter endovascular embolization has been preferred for the treatment of peripancreatic bleeding pseudoaneurysms because of the high risk, ineffectiveness, and high mortality during surgery, and it represents a safe alternative to surgery $[4,6]$. These are the reasons, in fact, for the decision to perform endovascular embolization to treat our patient. In the case of pancreatic pseudoaneurysms, endovascular embolization represents either a temporizing measure to control rapid bleeding, allowing haemodynamic stabilization, or a definitive therapy $[1,31]$. In our case, endovascular embolization proved to be sufficient and clinically effective alone. The target of the endovascular approach to pseudoaneurysms is either to pack the aneurysm with embolic agents or to exclude the neck of the aneurysm from the circulation $[1,2,12,13]$. The embolization of the proximal and distal neck is the most commonly reported endovascular therapy in such cases, because it is necessary to exclude both in-flow and out-flow tracts to reduce the risk of anterograde and retrograde reperfusion [23]. Gelfoam and coils are the most common agents used for either of these approaches, but detachable balloons, polyvinyl alcohol (PVA), and liquid embolic agents are also described in the literature $[1,2,12,13,32]$. Coils, detachable balloons, and PVA are permanent embolic agents, while Gelfoam is temporary and is reabsorbed within a few weeks, and in this way, recanalization can occur if it is used alone $[1,32]$. In addiction, both Gelfoam and PVA can migrate towards the distal splenic artery circle, with the risk of subsequent splenic infarctions and abscess $[1,29]$. The most frequent and effective embolic agent described to embolize the inflow and out-flow tracts of the injured artery, allowing the successful exclusion of the aneurysm's neck, is represented by metallic coils, which in most cases are sufficient and effective alone, as happened in our case, in which we successfully employ onlyed metallic coils for embolization $[1,11,12]$. When embolization is performed, the weakness of the wall represents an additional problem to consider, because the fragility of the pseudoaneurysm may allow it to rupture during injection of contrast material or chosen embolic agents, when the catheter is wedged into the feeding vessel. In fact, a considerable increase in intra-aneurysmal pressure occurs when a notable amount of fluid is injected in a short time, such as during endovascular procedures. In this way, all the injecting pressure is transmitted to the aneurysm's wall, and consequently rupture is favoured $[4,6]$. Therefore, the advantage of transcatheter coil embolization, with which no high pressure or large amount of fluid is necessary during the procedure, is clear and shared $[4,33]$. During the procedure, it is often necessary to employ microcatheters for the cannulation of the splenic artery, especially if tortuous, and for the correct and safe distal release of coils. It is usually necessary, in addiction, to release multiple coils to be clinically effective [1]. In our patient, successful embolization required the employment of both a microcatheter, owing to splenic artery's tortuosity, to selectively catheterize the out-flow tract, and release and packing of multiple metallic coils. The reported successful employment of n-butyl-cyanoacrylate (NBCA), especially in neurovascular interventions, has led to its use for embolizations in other regions too [1]. NBCA is often used alone to occlude the main vessel, but it can also be helpful for the embolization of aneurysms for which it is not possible to release further coils and to prevent delayed migration of microcoils with non-target embolization $[1,34]$. In recent years, its employment in the embolization of splenic artery pseudoaneurysms has also been reported $[1,35]$. Stent grafts can also be used, and their employment is already described in the literature, because they allow one to preserve arterial patency and splenic artery circulation. Their main limitations are due to difficulties of deployment in a tortuous vessel, like the splenic artery in our case, and post-stenting thrombosis $[1,8,29,36]$. However, preservation of the splenic artery is not always necessary; in fact, the extensive collateral arterial supply to the spleen prevents infarction when the main splenic artery is occluded, even though infarction can rarely occur $[1,13]$. The reported rate of complications of endovascular embolization is between $3 \%$ and $18 \%$; the major complications are represented by acute exacerbation of chronic pancreatitis, pancreatic necrosis, splenic infarct and abscess formation, intestinal infarct, infection, fever, dislodgement and migration of embolic agents, haematoma or pseudoaneurysm at the percutaneous arterial entry site, and contrastinduced acute renal failure $[1,8,30,31,36,37]$. When splenic infarct occurs patients may need splenectomy due to the possibility of developing an abscess or septicaemia $[1,31,32]$. In our patient, the embolization of the splenic 
artery did not cause a significant decrease in arterial supply to the organ, thanks to the extensive collateral arterial system, and, in this way, the portion of subsequent ischaemic parenchyma was so small as to be clinically neglectable, allowing preservation of the spleen with its precious immunite function.

\section{Conclusions}

Splenic artery pseudoaneurysms are often due to pancreatitis, acute and chronic, especially in the presence of pseudocysts. Endovascular embolization is a minimally invasive, safe, and effective alternative treatment to surgery,

with lower morbidity and mortality and high success rates, allowing preservation of the spleen and its precious immune function.

\section{Conflict of interest}

The authors report no conflict of interest.

\section{References}

1. Venkatesh SK, Kumar S, Baijal SS, et al. Endovascular management of pseudoaneurysms of the splenic artery: experience with six patients. Australas Radiol 2005; 49: 283-288.

2. Mandel SR, Jaques PF, Mauro MA, Sanofsky S. Non-operative management of peripancreatic arterial aneurysms. A 10-year experience. Ann Surg 1987; 205: 126-128.

3. Stabile BE, Wilson SE, Debas HT. Reduced mortality from bleeding pseudocysts and pseudoaneurysms caused by pancreatitis. Arch Surg 1983; 118: 45-51.

4. Uflacker R, Diehl JC. Successful embolization of a bleeding splenic artery pseudoaneurysm secondary to necrotizing pancreatitis. Gastrointest Radiol 1982; 7: 379-382.

5. White A, Baum S, Buranasari S. Anenrysms secondary to pancreatitis. Am J Radiol 1976; 127: 393-396.

6. Lima JR, Jaques P, Mandeli V. Aneurysm rupture secondary to transcatheter embolization. Am J Radiol 1979; 132: 553-556.

7. Sugg SL, Gerndt SJ, Hamilton BJ, et al. Pseudoaneurysms of the intraparenchymal splenic artery after blunt abdominal trauma: a complication of nonoperative therapy and its management. J Trauma 1995; 39: 593-595

8. Yagmur Y, Akbulut S, Gumus S, Demircan F. Giant splenic artery pseudoaneurysm: a case report and literature review. Int Surg 2015; 100: 1244-1248.

9. Uyar IS, Okur FF, Akpinar BA, et al. Giant splenic artery aneurysm: a case report. Turk Gogus Kalp Damar Cerrahisi Dergisi 2013; 21: 799-802 [in Turkish].

10. Mishra PK, Saluja SS, Sharma AK, Pattnaik P. Management of splenic artery aneurysm associated with extrahepatic portal vein obstruction. Hepatobiliary Pancreat Dis Int 2012; 11: 330-333.

11. Tessier DJ, Stone WM, Fowl RJ, et al. Clinical features and management of the splenic artery pseudoaneurysm: case series and cumulative review of literature. J Vasc Surg 2003; 38: 969-974.

12. Golzarian J, Nicaise N, Deviere J, et al. Transcatheter embolization of pseudoaneurysms complicating pancreatitis. Cardiovasc Intervent Radiol 1997; 20: 435-440.

13. McDermott VG, Shlansky-Goldberg R, Cope C. Endovascular management of splenic artery aneurysms and pseudoaneurysms. Cardiovasc Intervent Radiol 1994; 17: 179-184.
14. Bakhos CT, McIntosh BC, Nukta FA, et al. Staged arterial embolization and surgical resection of a giant splenic artery aneurysm. Ann Vasc Surg 2007; 21: 208-210.

15. Aroor AR, Prakasha SR, U R, Attar NR. Multiple splenic artery aneurysms: a rare cause of extrahepatic portal hypertension and massive splenomegaly. J Clin Diagn Res 2014;8: MD01-MD02.

16. Yadav S, Sharma P, Singh PK, et al. Giant splenic artery aneurysm: a rare but potentially catastrophic surgical challenge. Int J Surg Case Rep 2012; 3: 533-536.

17. Ho MF, Chan YC, Cheng SW. Successful endovascular management of giant splenic artery aneurysms. Vascular 2013; 21: 317-322.

18. Tekola BD, Arner DM, Behm BW. Coil migration after transarterial coil embolization of a splenic artery pseudoaneurysm. Case Rep Gastroenterol 2013; 7: 487-491.

19. Schecter IM, Gordon HE, Passaro E. Massive hemorrhage of the celiac axis in pancreatitis. Am J Surg 1974; 128: 301-305.

20. Pagliariccio G, Catalini R, Giantomassi L, Angelini A. Management of pseudoaneurysm of the leg: is Color Doppler US enough? J Ultrasound 2010; 13: 61-65.

21. Corvino A, Catalano O, Corvino F, et al. Superficial temporal artery pseudoaneurysm: what is the role of ultrasound? J Ultrasound 2016; 19: 197-201.

22. Tufano A, Minelli R, Rossi E, et al. Inferior epigastric artery pseudoaneurysm secondary to port placement during a robot-assisted laparoscopic radical cystectomy. J Ultrasound 2020; doi: 10.1007/s40477020-00442-1 [Online ahead of print].

23. Corvino A, Sandomenico F, Setola SV, et al. Added value of contrastenhanced ultrasound (CEUS) with Sonovue ${ }^{\circledR}$ in the diagnosis of inferior epigastric artery pseudoaneurysm: report of a case and review of literature. J Ultrasound 2019; 22: 485-489.

24. Kumar SK, Oon OK, Horgan P, Leen E. Early diagnosis of intrahepatic pseudoaneurysm during radiofrequency ablation using contrastenhanced ultrasound. Malays J Med Sci 2015; 22: 58-62.

25. Borzelli A, Amodio F, Paladini A, et al. Successful endovascular treatment of a recurrent giant celiac artery aneurysm. Radiol Case Rep 2019; 14: 723-728.

26. Rathod J, Taori K, Dhomane S, et al. Endovascular embolisation of giant ruptured proximal splenic arterial pseudoaneurysm using microcoils \& glue: case report. Surg Sci 2011; 2: 290-293.

27. Cangiano G, Corvino F, Giurazza F, et al. Endovascular treatment of simultaneous iliac and superficial femoral arterial pseudoaneurysms after stenting procedure complications. Vasc Endovascular Surg 2019; 53: $160-164$. 
28. Giurazza F, Silvestre M, Cervo A, Maglione F. Endovascular treatment of a dissected celiac trunk aneurysm complicated with consequent pseudoaneurysm: primary treatment and treatment relapse after 5 years. Case Rep Vasc Med 2015; 2015: 291953. doi: 10.1155/2015/ 291953.

29. Al-Habbal Y, Christophi C, Muralidharan V. Aneurysms of the splenic artery - a review. Surgeon 2010; 8: 223-231.

30. Borzelli A, Paladini A, Giurazza F, et al. Successful endovascular embolization of an intralobar pulmonary sequestration. Radiol Case Rep 2017; 13: 125-129.

31. Stabile BE. Hemorrhagic complications of pancreatitis and pancreatic pseudocysts. In: Beger HG et al. (eds). The Pancreas. Blackwell Science, Oxford 1998; 606-613.

32. Salam TA, Lumsden AB, Martin LG, Smith RB III. Non-operative management of visceral aneurysms and pseudoaneurysms. Am J Surg 1992;164: 215-219.

33. Wallace S, Gianturco C, Anderson J, et al. Therapeutic vascular occlusion using steel coil technique: clinical applications. Am J Radiol 1976; 127: 381-387.

34. Lupattelli T, Garaci FG, Sandhu C, et al. Endovascular treatment of giant splenic aneurysm that developed after liver transplantation. Transpl Int 2003; 16: 756-760.

35. Kim BS, Do HM, Razavi M. N-Butyl cyanoacrylate glue embolization of splenic artery aneurysms. J Vasc Interv Radiol 2004; 15: 91-94.

36. Boudghene F, L'Hermine C, Bigot JM. Arterial complications of pancreatitis: diagnostic and therapeutic aspects in 104 cases. J Vasc Interv Radiol 1993; 4: 551-558.

37. Miao YD, Ye B. Intragastric rupture of splenic artery aneurysms: three case reports and literature review. Pak J Med Sci 2013; 29: 656-659. 